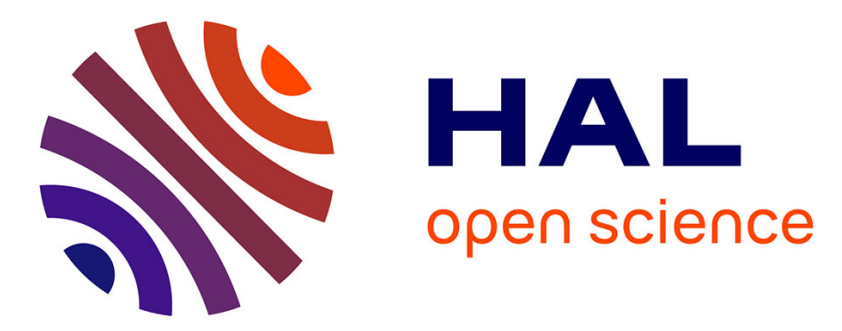

\title{
Propriétés statiques d'une chaîne isotrope alternée de spins quantiques $1 / 2$ et de spins classiques
}

\author{
J. Seiden
}

\section{To cite this version:}

J. Seiden. Propriétés statiques d'une chaîne isotrope alternée de spins quantiques

$1 / 2$ et de spins classiques. Journal de Physique Lettres, 1983, 44 (23), pp.947-952. 10.1051/jphyslet:019830044023094700 . jpa-00232286

\section{HAL Id: jpa-00232286 https://hal.science/jpa-00232286}

Submitted on 1 Jan 1983

HAL is a multi-disciplinary open access archive for the deposit and dissemination of scientific research documents, whether they are published or not. The documents may come from teaching and research institutions in France or abroad, or from public or private research centers.
L'archive ouverte pluridisciplinaire HAL, est destinée au dépôt et à la diffusion de documents scientifiques de niveau recherche, publiés ou non, émanant des établissements d'enseignement et de recherche français ou étrangers, des laboratoires publics ou privés. 
Classification

Physics Abstracts

$75.10 \mathrm{~J}$

\title{
Propriétés statiques d'une chaîne isotrope alternée de spins quantiques $1 / 2$ et de spins classiques
}

\author{
J. Seiden \\ Institut d'Electronique Fondamentale (*), Université de Paris XI, Bât. 220, 91405 Orsay, France
}

(Reçu le 18 juillet 1983, accepté le 7 octobre 1983)

\begin{abstract}
Résumé. - Les propriétés magnétiques statiques d'une chaîne isotrope formée en un ordre alterné de spins quantiques $1 / 2$ et de spins classiques sont déterminées analytiquement en toute rigueur. La susceptibilité théorique est un bon accord avec les mesures de Verdaguer sur le composé $\mathrm{CuMn}\left(\mathrm{S}_{2} \mathrm{C}_{2} \mathrm{O}_{2}\right)_{2}\left(\mathrm{H}_{2} \mathrm{O}\right)_{7,5}$.
\end{abstract}

\begin{abstract}
The static magnetic properties of an isotropic chain containing in an alternating order quantum 1/2 spins and classical spins are calculated rigorously and given analytically. The theoretical susceptibility is in fair agreement with the experimental values of Verdaguer for $\operatorname{CuMn}\left(\mathrm{S}_{2} \mathrm{C}_{2} \mathrm{O}_{2}\right)_{2}\left(\mathrm{H}_{2} \mathrm{O}\right)_{7.5}$.
\end{abstract}

\section{Introduction.}

Récemment, Gleizes et Verdaguer [1] ont réussi à synthétiser le composé $\mathrm{CuMn}\left(\mathrm{S}_{2} \mathrm{C}_{2} \mathrm{O}_{2}\right)_{2}$ $\left(\mathrm{H}_{2} \mathrm{O}\right)_{7,5}$, dont l'analyse structurale a montré que les ions $\mathrm{Cu}$ et $\mathrm{Mn}$ s'alignent le long de chaînes où ils se succèdent en un ordre régulier alterné $\mathrm{Cu}-\mathrm{Mn}-\mathrm{Cu}-\mathrm{Mn}-.$. Etudiant la susceptibilité $\chi(T)$ de cette substance en fonction de la température $T$, ils ont montré que la quantité $T \chi(T)$ passait par un minimum. Du point de vue théorique $\chi$ a été évaluée pour une chaîne alternée spin classique-spin quantique $1 / 2$ par Blöte [2], mais ses résultats, reposant sur des approximations, ne rendent pas compte des résultats expérimentaux. Nous avons montré [3] en utilisant l'hamiltonien isotrope (1) défini plus bas que le calcul de $\chi(T)$ pouvait être ramené à la diagonalisation d'une matrice d'ordre six et obtenu, en l'effectuant numériquement, un excellent accord avec la courbe expérimentale, prouvant ainsi que le couplage isotrope (1) entre ions $\mathrm{Cu}$ et $\mathrm{Mn}$ représente une base correcte pour l'étude des propriétés statiques de ce composé au-dessus de sa température de Néel. Nous montrons ici qu'il est possible d'évaluer analytiquement en toute rigueur :

a) l'énergie libre en champ nul $F$;

b) les corrélations statiques à deux spins (classique-classique, quantique-classique, quantiquequantique).

Nous en déduirons la susceptibilité $\chi$ en champ nul, en bon accord avec l'expérience.

(*) Laboratoire associé au C.N.R.S. 


\section{Hamiltonien.}

L'hamiltonien de la chaîne alternée isotrope est de la forme

$$
\mathscr{H}=\sum_{i=1}^{N-1} \mathscr{H}_{i}, \quad H_{i}=J\left[\mathbf{S}_{i}+\mathbf{S}_{i+1}\right] \mathbf{s}_{i} .
$$

Les $\mathbf{s}_{i}$, spins des ions $\mathrm{Cu}$, seront traités comme des opérateurs quantiques avec $s=1 / 2$. Par opposition, les $S_{i}$, spins des ions $\mathrm{Mn}$, seront considérés comme des vecteurs de longueur $S$. Chaque $s_{i}$ n'est couplé qu'à ses deux plus proches voisins $S_{i}$ et $S_{i+1}$. Tous les $\mathscr{H}_{i}$ commutent entre eux.

\section{Energie libre.}

La fonction de partition de la chaîne est

$$
Z=\left(\frac{2 S+1}{4 \pi}\right)^{N} \int \mathrm{d} \Omega_{1} \ldots \mathrm{d} \Omega_{N} z_{1,2} z_{2,3} \ldots z_{i, i+1} \ldots z_{N-1, N}
$$

avec

$$
\begin{gathered}
z_{i, i+1}=\exp \left(\frac{-\beta J S_{i, i+1}}{2}\right)+\exp \left(\frac{\beta J S_{i, i+1}}{2}\right) \\
. \mathbf{\delta}_{i, i+1}=\mathbf{S}_{i}+\mathbf{S}_{i+1}, \quad \mathbf{S}_{i, i+1}=\left|\mathbf{S}_{i, i+1}\right|=S \sqrt{2\left(1+\cos \theta_{i, i+1}\right)}
\end{gathered}
$$

$\theta_{i, i+1}$ est l'angle de $S_{i}$ avec $S_{i+1}$. Il y a $N$ spins classiques et $N-1$ spins quantiques. Dans (3), on a sommé sur les degrés de liberté quantiques et dans (2), intégré sur les degrés de liberté classiques. $S, \theta_{i}, \varphi_{i}$ sont les coordonnées polaires de $S_{i}$ et de $\mathrm{d} \Omega_{i}=\sin \theta_{i} \mathrm{~d} \theta_{i} \mathrm{~d} \varphi_{i}$.

On développera (3) en série de polynômes de Legendre

$$
z_{i, i+1}=z\left(\theta_{i, i+1}\right)=\sum_{l=0}^{\infty} a_{l} P_{l}\left(\cos \theta_{i, i+1}\right)
$$

d'où, d'après un calcul de Fisher [4]

$$
Z=(2 S+1)^{N}\left(a_{0}\right)^{N-1}, \quad F=-\frac{1}{\beta} \log Z .
$$

\section{Corrélation à deux spins.}

On s'intéresse aux trois types de corrélations :

$$
\begin{aligned}
\left\langle S_{i}^{z} S_{i+p}^{z}\right\rangle=\left(\frac{2 S+1}{4 \pi}\right)^{N} & \frac{1}{Z} \int \mathrm{d} \Omega_{1} \ldots \mathrm{d} \Omega_{N} z_{1,2} \ldots z_{i-1, i} S \cos \theta_{i} \times \\
& \times z_{i, i+1} \ldots z_{i+p-1, i+p} S \cos \theta_{i+p} z_{i+p, i+p+1} \\
& \times \cdots z_{N-1, N} . \\
\left\langle s_{i}^{z} S_{i+p}^{z}\right\rangle=\left(\frac{2 S+1}{4 \pi}\right)^{N} & \frac{1}{Z} \int \mathrm{d} \Omega_{1} \ldots \mathrm{d} \Omega_{N} z_{1,2} \ldots z_{i-1, i} y_{i, i+1} \times \\
& \times z_{i+1, i+2} \ldots z_{i+p-1, i+p} S \cos \theta_{i+p} z_{i+p, i+p+1} \\
& \times \cdots z_{N-1, N} .
\end{aligned}
$$




$$
\begin{aligned}
\left\langle s_{i}^{z} s_{i+p}^{z}\right\rangle=\left(\frac{2 S+1}{4 \pi}\right)^{N} & \frac{1}{Z} \int \mathrm{d} \Omega_{1} \ldots \mathrm{d} \Omega_{N} z_{1,2} \ldots z_{i-1, i} y_{i, i+1} \times \\
& \times z_{i+1, i+2} \ldots z_{i+p-1, i+p} y_{i+p, i+p+1} \\
& \times z_{i+p+1, i+p+2 \ldots z_{N-1, N}}
\end{aligned}
$$

avec

$$
y_{i, i+1}=\operatorname{Trace}\left\{s_{i}^{z} \exp \left(-\beta J \mathbf{s}_{i, i+1} \mathbf{s}_{i}\right)\right\}
$$

\section{Evaluation des corrélations classiques.}

On a

$$
a_{0}=\frac{1}{2} \int_{0}^{\pi} z(\theta) \sin \theta \mathrm{d} \theta, \quad a_{1}=\frac{3}{2} \int_{0}^{\pi} z(\theta) \cos \theta \sin \theta \mathrm{d} \theta .
$$

On fera dans (11) les changements de variable $\theta / 2=\alpha$, puis $\cos \alpha=x$, utilisant

$$
\left.\begin{array}{c}
\int u \operatorname{ch} u \mathrm{~d} u=u \operatorname{sh} u-\operatorname{ch} u \\
\int u^{3} \operatorname{ch} u \mathrm{~d} u=\left(u^{3}+6 u\right) \operatorname{sh} u-\left(3 u^{2}+6\right) \operatorname{ch} u .
\end{array}\right\}
$$

On trouve finalement :

$$
\left.\begin{array}{l}
a_{0}=4\left[\gamma^{-1} \operatorname{sh} \gamma-\gamma^{-2} \operatorname{ch} \gamma+\gamma^{-2}\right] \\
a_{1}=12\left[\left(\gamma^{-1}+12 \gamma^{-3}\right) \operatorname{sh} \gamma-\left(5 \gamma^{-2}+12 \gamma^{-4}\right) \operatorname{ch} \gamma-\gamma^{-2}+12 \gamma^{-4}\right]
\end{array}\right\}
$$

avec $\gamma=\beta J S=J S / k_{\mathrm{B}} T$. On en déduit d'abord l'énergie libre (6) puis

$$
\left\langle S_{i}^{z} S_{i+p}^{z}\right\rangle=\delta^{p} \frac{S^{2}}{3}
$$

avec

$$
\delta=\frac{a_{1}}{3 a_{0}}
$$

\section{Evaluation des corrélations renfermant des spins quantiques.}

Pour évaluer (10), on prendra comme base les états propres $|m\rangle$ de $s^{2}: s^{2}|m\rangle=m|m\rangle$, d'où :

$$
y=\sum_{m} m \sum_{\mu}\langle m \mid \mu\rangle\langle\mu|\exp (-\beta J \mathbf{s s})| \mu\rangle\langle\mu \mid m\rangle
$$

où $|\mu\rangle$ sont les états propres de la projection de s sur 8: Les $\langle m \mid \mu\rangle$ sont les éléments de la matrice $D^{1 / 2}(\psi)$, représentation spinorielle du groupe des rotations introduite par Wigner [5].

$$
D^{1 / 2}(\psi)=\left(\begin{array}{rr}
\cos \frac{\psi}{2} & -\sin \frac{\psi}{2} \\
\sin \frac{\psi}{2} & \cos \frac{\psi}{2}
\end{array}\right)
$$


où $\psi$ est l'angle de $\boldsymbol{S}$ avec $\mathrm{O} z$. Ainsi, avec $s=1 / 2$,

$$
\left.\begin{array}{c}
y=-2 s \cos \psi \operatorname{sh}\left(\frac{\beta J \mathrm{~S}}{2}\right) \\
\cos \psi_{i, i+1}=\frac{S_{i, i+1}^{z}}{S_{i, i+1}}=\frac{S\left(\cos \theta_{i}+\cos \theta_{i+1}\right)}{S_{i, i+1}} .
\end{array}\right\}
$$

Nous développons sh $\left(\frac{\beta J S}{2}\right) / \mathrm{S}$ en série de polynômès de Legendre

$$
\frac{S \operatorname{sh}\left(\frac{\beta J S_{i, i+1}}{2}\right)}{S_{i, i+1}}=\sum_{l=0}^{\infty} b_{l} P_{l}\left(\cos \theta_{i, i+1}\right) .
$$

Les coefficients $b_{0}$ et $b_{1}$ sont obtenus par une méthode identique à celle utilisée pour déterminer $a_{0}$ et $a_{1}$. A l'aide de

$$
\int u^{2} \operatorname{sh} u \mathrm{~d} u=\left(u^{2}+2\right) \operatorname{ch} u-2 u \operatorname{sh} u
$$

il vient

$$
\left.\begin{array}{l}
b_{0}=\gamma^{-1}(\operatorname{ch} \gamma-1) \\
b_{1}=3\left[\left(\gamma^{-1}+4 \gamma^{-3}\right) \operatorname{ch} \gamma-4 \gamma^{-2} \operatorname{sh} \gamma+\gamma^{-1}-4 \gamma^{-3}\right] .
\end{array}\right\}
$$

Portons maintenant (18) et (19) dans (8) et (9).

Introduisant :

$$
\Lambda=2\left[\frac{b_{1}}{3 a_{0}}+\frac{b_{0}}{a_{0}}\right]
$$

Il vient finalement

$$
\begin{aligned}
& \left\langle s_{i}^{z} S_{i+p}^{z}\right\rangle=-\Lambda \delta^{p-1} \frac{s S}{3}, \quad p>0 \\
& \left\langle s_{i}^{z} s_{i+p}^{z}\right\rangle=\Lambda^{2} \delta^{p-1} \frac{s^{2}}{3}, \quad p>0 \\
& \left\langle s_{i}^{z} s_{i}^{z}\right\rangle=\frac{s(s+1)}{3}
\end{aligned}
$$

On remarque que les corrélations quantiques $\left\langle s_{i}^{z} s_{i+p}^{z}\right\rangle$ pour $p \neq 0$ sont proportionnelles à $s^{2}$ et non pas à $s(s+1)$ (on verra que pour $\left.T \rightarrow 0, \Lambda^{2} \delta^{p-1} \rightarrow 1\right)$. On vérifie également que toutes les corrélations entre un spin situé en $i$ et un spin situé en $i+p$ varient comme $\delta^{p}$ en fonction de leur distance $p$. Examinons maintenant deux cas limites :

a) Basses températures $\beta|J| S \gg 1$. On trouve ici

$$
\delta \simeq 1-\frac{4}{\beta|J| S}, \quad \Lambda \simeq \frac{J}{|J|}\left(1-\frac{1}{\beta|J| S}\right) .
$$


b) Hautes températures $\beta|J| S \ll 1$. On trouve alors

$$
\delta \simeq \frac{(\beta J S)^{2}}{12}, \quad \Lambda \simeq \frac{\beta J S}{2}\left(1-\frac{5}{36}(\beta J S)^{2}\right) .
$$

\section{Susceptibilité magnétique.}

Désignons par $g_{s}$ et $g_{s}$ les facteurs $g$ respectivement des spins classiques et quantiques. La susceptibilité en champ nul est

$$
\chi=\beta \mu_{\mathrm{B}}^{2} \sum_{i, j}\left\langle\left(g_{S} S_{i}^{z}+g_{s} s_{i}^{z}\right)\left(g_{S} S_{j}^{z}+g_{s} s_{j}^{z}\right)\right\rangle .
$$

Utilisant les corrélations (14) et (23), il vient :

$$
\begin{aligned}
\chi=\frac{N \beta \mu_{\mathrm{B}}^{2}}{3}\left[g_{S}^{2} S^{2}\left(\frac{S+1}{S}+2 \frac{\delta}{1-\delta}\right)-4 g_{S} g_{s} \Lambda s S\right. & \frac{1}{1-\delta}+ \\
& \left.+g_{s}^{2}\left(s(s+1)+2 \Lambda^{2} s^{2} \frac{1}{1-\delta}\right)\right]
\end{aligned}
$$

Dans (27), nous avons remplacé la self-corrélation $\left\langle S_{i}^{z} S_{i}^{z}\right\rangle$ par sa valeur quantique $S(S+1) / 3$, ce qui fournit pour $\chi(T \rightarrow \infty)$ la limite quantique correcte.

Aux basses températures où $\beta J S \gg 4,(27)$ devient

$$
\chi \simeq \frac{N \beta \mu_{\mathrm{B}}^{2}}{3} \frac{\beta J S}{2}\left(g_{s} S-\frac{J}{|J|} g_{s} s\right)^{2}
$$

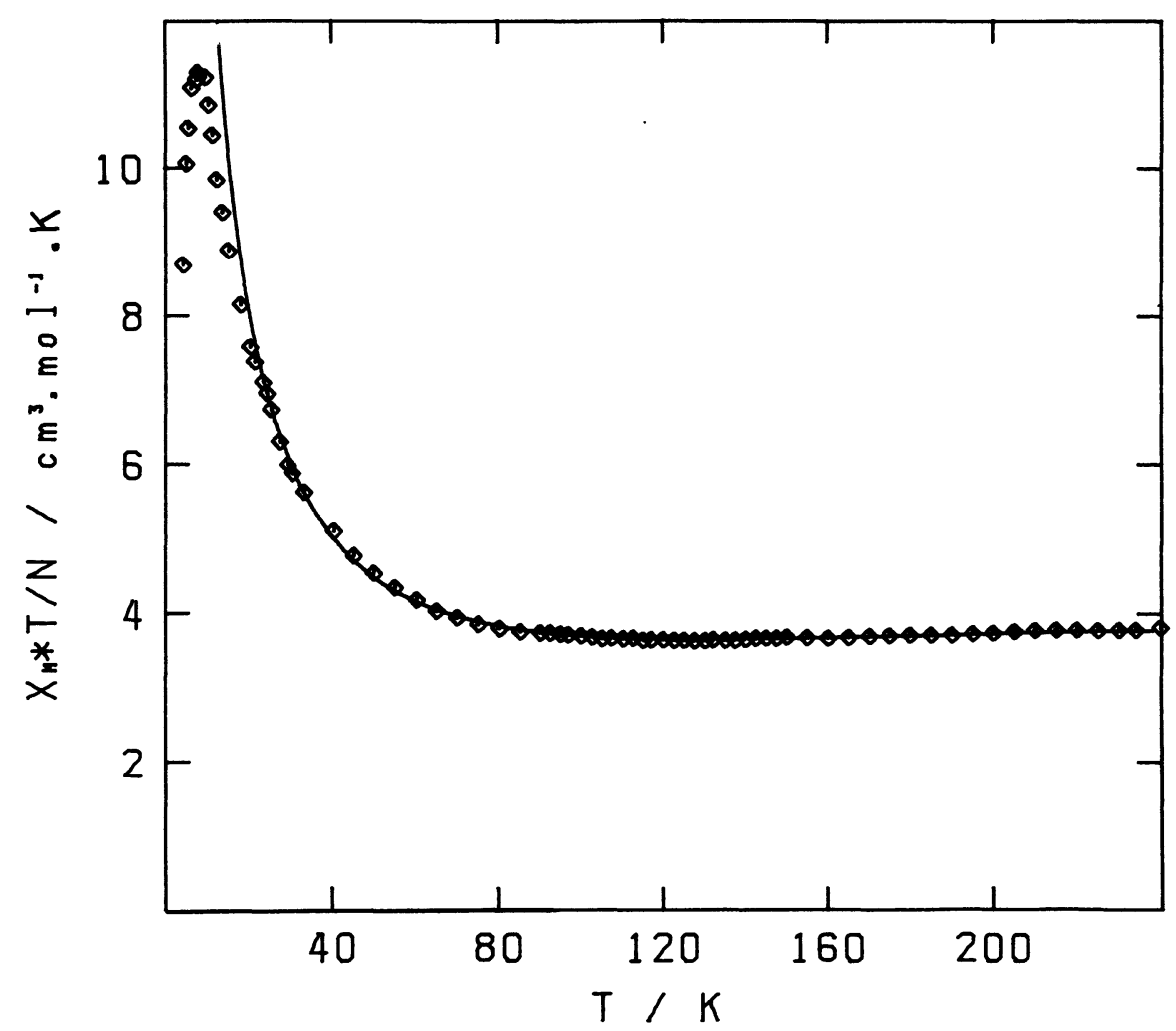

Fig. 1. - Expérience [Experiment]; — Théorie [Theory]. 
c'est-à-dire $\chi \propto T^{-2}$. Lorsque $J>0$ [couplage antiferromagnétique entre les $\mathbf{s}$ et les $\mathbf{S}$, la quantité $T \chi(T)$ passe par un minimum à une température $T_{\mathrm{m}}$ située généralement dans la région où $\beta J S<1]$. Pour $S \gg 1, T_{\mathrm{m}}$ peut être déterminée analytiquement : posant $\rho=g_{\mathrm{s}} / g_{S}$, on trouve

$$
\frac{J S}{k_{\mathrm{B}} T_{\mathrm{m}}} \simeq \frac{\left[\left(S^{2}+3 \rho^{2} s^{2}\right)^{2}+24(\rho s S)^{2}\right]^{1 / 2}-\left(S^{2}+3 \rho^{2} s^{2}\right)}{2 \rho S S} .
$$

\section{Comparaison avec l'expérience.}

Sur la figure, on a porté $T \chi(T)$ en fonction de $T$. Les carrés représentent les valeurs expérimentales de Verdaguer relatives à $\mathrm{CuMn}\left(\mathrm{S}_{2} \mathrm{C}_{2} \mathrm{O}_{2}\right)_{2}\left(\mathrm{H}_{2} \mathrm{O}\right)_{7,5}$, on a $T_{\mathrm{m}}=130 \mathrm{~K}$. Le trait continu est calculé à l'aide de (27) avec $J \simeq 59,7 \mathrm{~K}, g_{s}=g_{s} \simeq 1,9$. En dessous de $20 \mathrm{~K}$, les valeurs théoriques de $\chi(T)$ croissent plus vite que les valeurs mesurées du fait qu'alors, les interactions entre chaînes, négligées dans notre calcul, interviennent et induisent finalement une transition de phase à 7,5 $\mathrm{K}$.

L'excellent accord avec l'expérience pour $T>20 \mathrm{~K}$ ne doit cependant pas faire illusion puisque nous avons fait abstraction de toutes les anisotropies ainsi que du caractère quantique des $S$. Une discussion approfondie paraîtra ultérieurement [3].

\section{Remerciements.}

Je remercie M. Verdaguer qui a tracé la figure, ainsi que J. P. Renard et P. Veillet pour des discussions.

\section{Bibliographie}

[1] Gleizes, A. et Verdaguer, M., J. Amer. Chem. Soc. 103 (1981) 7373.

[2] BLÖte, H. W. J., J. Appl. Phys. 50 (1979) 7401.

[3] Gleizes, A., Verdaguer, M., Renard, J. P. et Seiden, J., Nato Advanced Institute, Castiglione 1983, et à paraître. M. Verdaguer, M. Julve, A. Michalowicz et $\mathrm{O}$. Kahn, à paraître.

[4] Fisher, M. E., Am. J. Phys. 32 (1964) 343.

[5] Wigner, E., Group Theory (Academic Press, N.Y.) 1959. 